\title{
Improving of Compound Modulation Signal Parameter Estimation Method
}

\author{
Ye Tao, Liping Wang, Ying Mu \\ Department of Electronic and Information Engineering LanZhou Institute of Technology LanZhou, China \\ Department of foreign languages LanZhou Institute of Technology LanZhou, China \\ Department of Electronic and Information Engineering LanZhou Institute of Technology LanZhou, China \\ 543627208@qq.com,44863332@qq.com, Taoye_666@126.com
}

\begin{abstract}
Modern communication commonly uses the complex modulation signal in order to achieve Anti-interference. Most current signal parameter estimation uses the hypothesis of some of the priori information known, which has some special features. This paper describes the design of a low SNR parameter estimation method of code width, cell bandwidth, PN code number, which transforms the signal with short-time Fu Liye. And then the method estimates the output signal by three order cumulation and achieves the parameter estimation by using the signal Radon conversion plans. The simulation results show that the method can live without prior knowledge, and has universal advantages .
\end{abstract}

Keywords- Complex Modulation Signal; Parameter Estimation; Higher-Order Cumulant

\section{INTRODUCTION}

The modern communication improves the anti-interference and confidentiality through transmitting low power complex modulated wideband signal. The Pseudo -linear frequency modulation combined signal which has good resolution of distance and velocity is universally applied by signal system,. Most Parameter estimate methods at present use the assumptions that information is partially known, so they are not suitable for low SNR signal parameter estimation [1]. Some methods require the pseudo random code sequence or [2] known code width and

carrier frequency, [3] while there is an estimation algorithm based on signal parameters of the Wigner distribution, it is seriously influenced by the cross terms of time-frequency plane.

This paper makes up disadvantages of the existing estimation methods. Firstly, it analyzes the characteristics of PN code combined with linear frequency modulation signal, and then it transforms the signal with short-time Fu Liye, applying the higher order cumulant restraining Gauss noise signal to the filter output of short-time estimation. Finally the main parameters' estimation of signal is obtained on the bases of on time-frequency graph transformation.

\section{PSEUdO - LiNEAR FREQUENCY MOdULATED SignAL}

Pseudo code phase modulation signals with linear frequency-modulated signal constitutes a pseudo codes-linear frequency-modulated signal in time domain:

$$
\begin{aligned}
u(t) & =u_{l f m}(t) * u_{p}(t) \\
& =\left[\frac{1}{\sqrt{T}} \operatorname{rect}\left(\frac{t}{T}\right) e^{j \pi k t^{2}}\right] *\left[\frac{1}{\sqrt{P}} \sum_{n=0}^{P-1} c_{n} \delta(t-n T)\right](1) \\
& =\frac{1}{\sqrt{P T}} \sum_{n=0}^{P-1} c_{n} \operatorname{rect}\left(\frac{t-n T}{T}\right) e^{j \pi k(t-n T)^{2}}
\end{aligned}
$$

Where:T is the pulse width of the LFM signal,P is the pseudo code sequence length, $\mathrm{K}$ is the slope of frequency modulation LFM signal, $C_{n}$ is the pseudo code sequence coefficient. The time-frequency relationship shown in figure 1 .

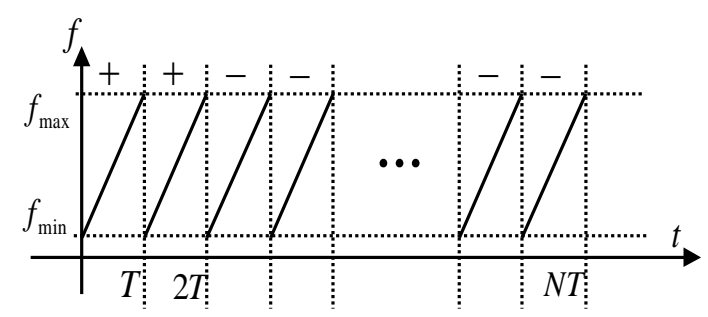

Figure 1. pseudo code LFM signal time frequency relationship

\section{ESTIMATION METHOD OF PSEUDO RANDOM \\ CODE - THE PARAMETERS OF LINEAR FREQUENCY MODUlATED SIGNAL}

\section{A. Short term Fu Liye transform}

Short-time Fourier transform (STFT) basic idea: using the superposition of a series of short-time stationary signal over non-stationary signal, implemented by time-domain-wide windowed short time and use the panning parameter to extend to the entire domain. This method is simple and clear, there is no crossover, you can resolve some problems in signal analysis.

Similar to STFT, short frequency Fu Liye transform (SFFT) for:

$$
S F F T_{\hat{s}}(t, \omega)=\frac{1}{2 \pi} \int_{-\infty}^{\infty} \hat{s}(\theta) \hat{h}(\omega-\theta) e^{j \theta t} d \theta
$$




\section{B. High order cumulant}

High order cumulants can suppress the Gauss noise, improve the parameter estimation and signal detection performance. On a zero mean signal, estimate three order cumulant segment, the short-time estimation for:

$$
\hat{c}_{3 x}\left(\tau_{1}, \tau_{2} ; i\right)=\frac{1}{S_{2}-S_{1}+1} \sum_{n=S_{1}}^{S_{2}} X_{i}(n) X_{i}\left(n+\tau_{1}\right) X_{i}\left(n+\tau_{2}\right)
$$

Among them:

$$
\begin{aligned}
& x_{i}(n)=\left\{\begin{array}{c}
x(n) w(n-i), i-K \leq n \leq i+K \\
0, \text { others }
\end{array}\right. \\
& S_{1}=\max \left\{i-K, i-K-\tau_{1}, i-K-\tau_{2}\right\} \\
& S_{2}=\min \left\{i+K, i+K-\tau_{1}, i+K-\tau_{2}\right\}
\end{aligned}
$$

The three order cumulant diagonal slice of Gauss signal can reduce the complexity and Gauss noise. Figure 2 is the signal to noise ratio for the modulation output $0 \mathrm{~dB}$, figure 3 is the output of the three order cumulant diagonal slice of the short-time estimation after contrast can be seen, this method has good effect on noise suppression.

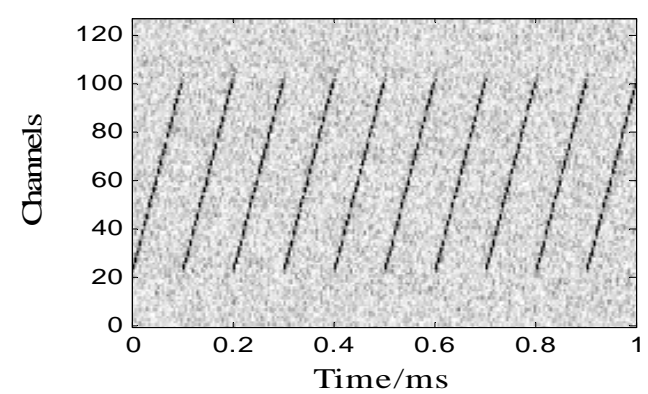

Figure 2 .Frequency diagram of polyphase filter bank output(0dB)

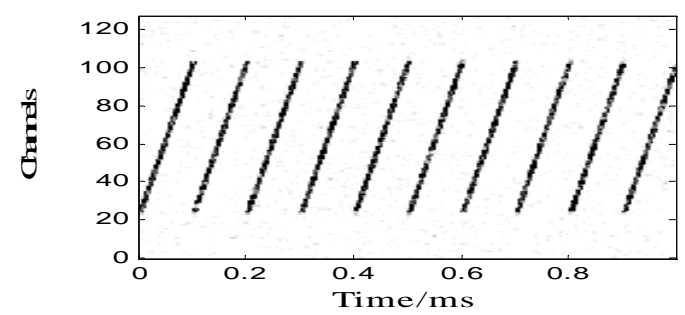

Figure 3. Three order cumulant estimator output

\section{Algorithm for signal parameter estimation process}

Based on the above analysis, In this paper the polyphase filter group and high order cumulant based on signal processing procedure is as follows:

(1) By means of a polyphase filter short frequency $\mathrm{Fu}$ Liye transform and signal detection, to obtain the required signal time-frequency distribution $\operatorname{SFFT}_{\hat{x}}(t, \omega)$

(2) The sub polyphase filter output signal processing,the three order cumulant diagonal slice short-time estimation, thus effectively restrain Gauss noise, The phase of the signal time-frequency distribution $\rho_{3 x}(t, \omega)$.

(3)The preceding phase output signal envelope detector, signal time-frequency map $\left|\rho_{3 x}(t, \omega)\right|$.

(4) Signals using time-frequency map parameter information, extracts the signal parameters, respectively.

\section{ANALYSIS OF THE EXPERIMENTAL SIMULATION AND ESTIMATION PERFORMANCE}

The root mean square error (SNR) as a measure of effectiveness standard verification algorithm. Technology and method in the reference, can better show the effectiveness of the method.

FM signal carrier in the simulation of $16 \mathrm{MHz}$, frequency range $6 \mathrm{MHz} \sim 26 \mathrm{MHz}$, carries on the analysis from 10 digit GOLD code, the total length of the signal $1 \mathrm{~ms}$. The zero mean white Gauss noise, the signal-to-noise ratio varied from $-12 \mathrm{~dB}$ to $0 \mathrm{~dB}$, the Monte Carlo test was 1000 times than each signal to noise. The composite signal pulse width, pseudo code number, bandwidth and the frequency estimation results are given, the simulation results in Figure 4 and Figure 5.

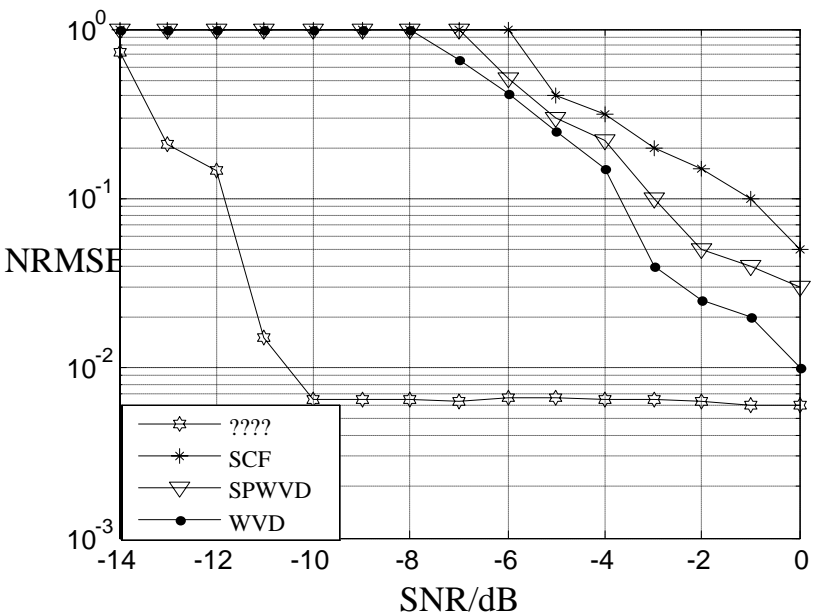

Figure 4. estimation of symbol-width $\mathrm{T}$ 


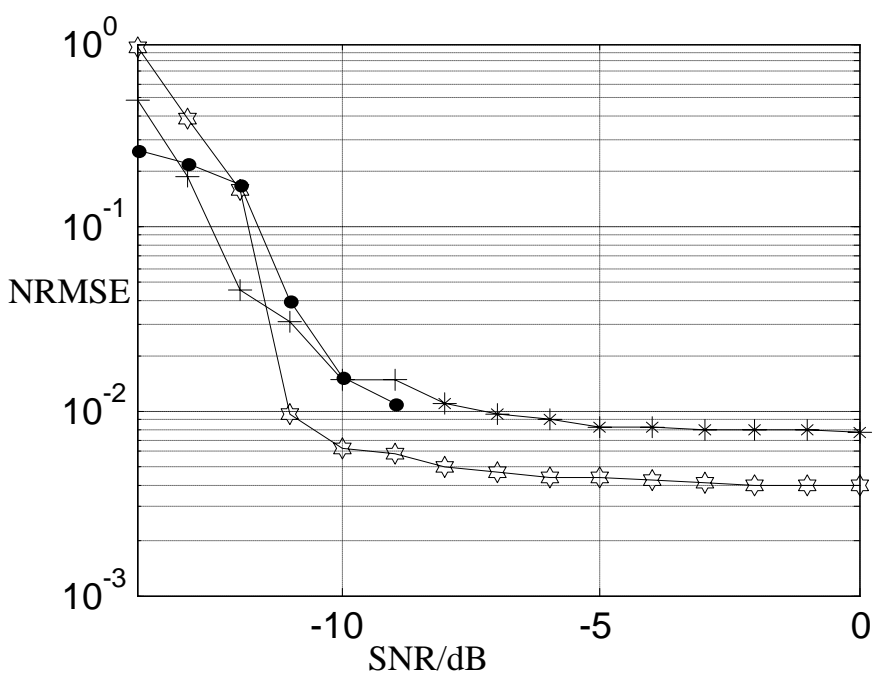

Figure 5 Bandwidth, number of pseudo-random code, start and end frequencies estimation

Performance estimation of code width is shown in Figure 5 , when the SNR is lower than $0 \mathrm{~dB}$, the estimation precision are higher than those of other methods. Figure 6 shows the initial frequency and the cutoff frequency, pseudo code number and signal bandwidth estimation, it can be seen from the figure, when the SNR is larger than $-10 \mathrm{~dB}$, the estimation accuracy is higher.

\section{SUMMARY}

Higher order cumulative volume has a better function of inhibition Gaussian noise; the more phase filter method is simple and has a high operation efficiency, which can improve letter noise rate and divide signals according to frequency domination. So this paper combines the two together, gets complete Shi frequency matrix signal, achieves signal parameter extraction of frequency domination, offers the estimated methods of pseudo yards-linear FM signal of starting frequency, as frequency, bandwidth, and pseudo yards number. The simulating, testing procedures and results in Gaussian environment show that this method of high precision, high speed, is a pseudo code-effective method for linear frequency modulation combined modulated signal parameter extraction. In the signal modulation, transmission, analysis and processing of the process, It has good application value.

\section{REFERENCES}

[1] Zhang Jiafen,He Xiao Dong, Tang Bin, "telecommunication technology fast parameter estimation of LFM signal,”The method of pseudo code , 2009,49 pp. 100-105

[2] Tao Jianyi, "LPI radar signal detection, analysis and classification of technical overview of, " electronic reconnaissance and jamming, 2004 (4) pp. 1-4

[3] Jin Yan, "LPI signal detection and parameter estimation of cyclostationarity ," Xi'an Electronic and Science University, 2008.

[4] Xiong Gang, Zhao Huichang, Wang Lijun, “ Reconnaissance signal recognition of PN code carrier frequency spectrum correlation method (I) / (II): pseudo code modulation recognition and parameter estimation of carrier frequency modulation signal," Journal of electronics and information technology, 2005,27 (7) pp.1081-1092. 